\author{
Series A
}

I. MATHEMATICA

593

\title{
DIE PROJEKTIVE, ABSOLUTKONVEXE TENSORPRODUKTLIMITIERUNG
}

VON

STEN BJON

HELSINKI 1974

S U O M A A INEN TIEDEAKATEMIA

doi:10.5186/aasfm.1975.593 
Copyright (C) 1974 by Academia Scientiarum Fennica

ISSN 0066-1953

ISBN 951-41-0209-6

Vorgelegt am 13. Mai 1974 


\section{Einleitung}

Die projektive, absolutkonvexe Tensorproduktlimitierung $\Lambda \otimes_{k} \Lambda^{\prime}$ auf dem Tensorprodukt $E \otimes F$ zweier absolutkonvexen Limesvektorräume $(E, A)$ und $\left(F, \Lambda^{\prime}\right)$ (vgl. [3]) wird definiert als die feinste absolutkonvexe Limitierung auf $E \otimes F$ für die die kanonische Abbildung $E \times F \rightarrow E \otimes F$ an der Stelle $(0,0)$ stetig ist. Die grundlegenden Eigenschaften dieser Tensorproduktlimitierung werden hergeleitet. In allen Untersuchungen verwenden wir die Charakterisierung der absolutkonvexen Limesvektorräume durch "Pseudonormen», die in [3] eingeführt wurde. Falls $(E, \Lambda)$ und $\left(F, \Lambda^{\prime}\right.$ ) ausgeglichene ([6]), absolutkonvexe Räume sind, so ist $\Lambda \otimes_{k} \Lambda^{\prime}$ ausgeglichen und gleich der feinsten absolutkonvexen Limitierung auf $E \otimes F$, für die $t$ global stetig ist. Mit Hilfe einer internen Charakterisierung der Marinescu-Räume, die mit einer in [11] gegebenen Charakterisierung eng zusammenhängt, wird gezeigt, dass $\Lambda \otimes_{k} \Lambda^{\prime}$ mit $\Lambda$ und $\Lambda^{\prime}$ eine Marinescu-Limitierung ist. Speziell folgt nun (siehe [8]), dass $\Lambda \otimes_{k} \Lambda^{\prime}$ gleich der projektiven Tensorprodukttopologie ([7]) ist, falls $\Lambda$ und $\Lambda^{\prime}$ lokalkonvexe Topologien sind.

Die Frage unter welchen Bedingungen $\Lambda \otimes_{k} \Lambda^{\prime}$ mit $\Lambda$ und $\Lambda^{\prime}$ separiert ist, haben wir nicht vollständig beantworten können (siehe Bemerkung im Anschluss an Satz 13).

\section{Begriffe und Bezeichnungen}

Im folgenden verstehen wir unter Vektorraum immer einen Vektorraum über dem Körper $\mathbf{K}$ der reellen oder komplexen Zahlen. $\mathbf{V}$ sei der Nullumgebungsfilter der natürlichen Topologie auf $\mathbf{K}$. Ein Filter $\mathcal{F}$ auf einem Vektorraum $E$ über $\mathbf{K}$ heisst nach [6] ausgeglichen (equable), falls gilt $\quad \mathbf{V} \cdot \mathcal{F}=\mathcal{F}$. Eine Vektorraumlimitierung $A$ auf $E$ heisst ausgeglichen, falls es für jedes $\mathcal{F} \in \Lambda 0$ ein ausgeglichenes $\mathscr{C}_{1} \in \Lambda 0$ mit $C_{1} \leq \mathcal{F}$ gibt. Die Vektorraumlimitierung $A$ heisst absolutkonvex, falls es zu jedem Filter $\mathcal{F} \in \Lambda 0$ einen Filter $\mathcal{C} \in \Lambda 0$ gröber als $\mathcal{F}$ mit einer Basis aus absolutkonvexen Mengen gibt.

Seien $E$ und $F \neq \phi$ Mengen und $\Omega$ eine Teilmenge der Menge aller Abbildungen $u: E \rightarrow F$. Die Evaluation ist die durch $\omega(u, x)=u(x)$ 
$(u \in \Omega, \quad x \in E)$ definierte natürliche Abbildung $\omega: \Omega \times E \rightarrow F$. Sei $Z$ eine weitere Menge. $\mathrm{Zu}$ jeder Abbildung $f: Z \rightarrow \Omega$ ist eine Abbildung $\alpha(f)=\tilde{f}=\omega \circ\left(f \times i d_{E}\right): Z \times E \rightarrow F$ assoziiert, wobei $i d_{E}$ die Identität auf $E$ bezeichnet.

Seien $E, E_{1}, \ldots, E_{n}$ Vektorräume und sei $\mathbf{R}^{+}$die Menge der nichtnegativen reellen Zahlen. Abbildungen $q: E \rightarrow \mathbf{R}^{+} \cup\{\infty\}$ mit den Eigenschaften

(a) $q(0)=0$

(b) $q(\lambda x)=|\lambda| q(x)$ $\lambda \neq 0, \lambda \in \mathbf{K}, \quad x \in E$

(c) $q(x+y)=q(x)+q(y)$ $x \in E, y \in E$

nennen wir Pseudonormen. Abbildungen $q: E_{1} \times \ldots \times E_{n} \rightarrow \mathbf{R}^{+} \cup\{\infty\}$, die die Pseudonormeigenschaften (b) und (c) für jedes "Argument» aufweisen, heissen $n$-Pseudonormen auf $E_{1} \times \ldots \times E_{n}$. Es seien $Q\left(E_{1}, \ldots, E_{n}\right)$ die Menge aller $n$-Pseudonormen auf $E_{1} \times \ldots \times E_{n}$ und $Q(E)$ die Menge aller Pseudonormen auf $E$. Die Ordnungsrelation $\leq$ auf $Q\left(E_{1}, \ldots, E_{n}\right)$ und $Q(E)$ sei die von der natürlichen Ordnung auf $\mathbf{R}^{+} \cup\{\infty\}$ induzierte punktweise Ordnung. Jedes Element $\left(q_{1}, \ldots, q_{n}\right) \in Q\left(E_{1}\right) \times \ldots \times Q\left(E_{n}\right)$ definiert durch $\left(q_{1} \ldots q_{n}\right)\left(x_{1}, \ldots, x_{n}\right)=q_{1}\left(x_{1}\right) \ldots q_{n}\left(x_{n}\right)$, wobei wir die Rechenregel $0 \cdot \infty=\infty$ verwenden, eine $n$-Pseudonorm

$$
q_{1} \ldots q_{n} \in Q\left(E_{1}, \ldots, E_{n}\right) .
$$

Für beliebige Teilmengen $M_{v} \in Q\left(E_{v}\right)(v=1, \ldots, n)$ und Filter $\psi_{v}$ auf $Q\left(E_{v}\right) \quad(v=1, \ldots, n)$ setzen wir

$$
M_{1} \ldots M_{n}=\left\{q^{\prime} q \leq q_{1} \ldots q_{n}, q_{v} \in M_{v} \text { für } v=1, \ldots, n\right\}
$$

und $\psi_{1} \ldots \psi_{n}=\left[\left\{M_{1} \ldots M_{n} \mid M_{v} \in \psi_{\nu}, v=1, \ldots, n\right\}\right]$.

Auf dem Raum $L\left(E_{1}, \ldots, E_{n} ; F\right)$ der $n$-linearen Abbildungen $w: E_{1} \times \ldots \times E_{n} \rightarrow F$ wird für beliebige Pseudonormen $q_{v} \in Q\left(E_{\nu}\right)$ $(v=1, \ldots, n), q^{\prime} \in Q(F)$, durch

$$
\begin{aligned}
\left(q^{\prime} / q_{1} \ldots q_{n}\right)(w)= & \sup _{q_{v}\left(x_{v}\right) \leq 1 ; \nu=1, \ldots, n} q^{\prime}\left(w\left(x_{1}, \ldots, x_{n}\right)\right)
\end{aligned}
$$

eine Pseudonorm $q^{\prime} / q_{1} \ldots q_{n}$ definiert.

Eine Teilmenge $M$ von $Q(E)$ heisst saturiert, falls die Pseudonormen $q_{1}$ und $q_{2}$ genau dann in $M$ sind, wenn $q_{1} \bigvee q_{2}=\sup \left(q_{1}, q_{2}\right)$ in $M$ ist. Ein Filter auf $Q(E)$ heisst saturiert, falls er eine Basis aus saturierten Mengen besitzt. Eine Teilmenge $M$ von $Q(E)$ heisst ausgeglichen, falls $\lambda M=M$ für jedes $\lambda>0$ ist.

Jede Pseudornom $q \in Q(E)$ definiert eine absolutkonvexe Teilmenge $U_{q}=\{x \mid q(x) \leq 1\}$ von $E$ und jede Teilmenge $M \subset Q(E)$ definiert einen 
Filter $\mathcal{F}_{M}=\left[\left\{U_{q} \mid q \in M\right\}\right]$ auf $E$. Nach [3] definiert ein Filter $\psi$ auf $Q(E)$ durch $\Lambda 0=\left[\left\{\mathcal{F}_{M} \mid M \in \psi\right\}\right], \quad \Lambda x=x+\Lambda 0$ eine absolutkonvexe Limitierung $\Lambda$ auf $E$, falls er die Bedingungen

(I) $\psi^{\lambda} \leq \psi$ für jedes $\lambda \neq 0, \quad \lambda \in \mathbf{K}$

(II) für jedes $x \in E$ gibt es ein $M \in \psi$, so dass $q(x)<\infty$ für jedes $q \in M$ ist

erfüllt. $\psi$ heisst dabei für $\Lambda$ definierend. Jede absolutkonvexe Limitierung kann in dieser Weise durch einen saturierten Filter auf $Q(E)$ mit den Eigenschaften (I) und (II) definiert werden. Ist die Limitierung diesüber ausgeglichen, so können wir einen definierenden Filter finden, der eine Basis aus ausgeglichenen, saturierten Mengen besitzt.

Seien $w \in L\left(E_{1}, \ldots, E_{n} ; F\right)$ und $q \in Q(F)$. Die Abbildung $q \circ w$ ist eine $n$-Pseudonorm auf $E_{1} \times \ldots \times E_{n}$, für die wir die Bezeichnung $q^{w}$ verwenden. Wir setzen weiter $M^{w}=\left\{q^{w} \mid q \in M\right\}$ und $\psi^{w}=\left[\left\{M^{w} \mid M \in \psi\right\}\right]$ für eine Teilmenge $M \subset Q(F)$ bzw. einen Filter $\psi$ auf $Q(F)$. Seien nun der saturierte Filter $\psi_{\nu}$ für die absolutkonvexe Limitierung $\Lambda_{v}$ auf $E_{v}$ $(v=1, \ldots, n)$ und der Filter $\psi^{\prime}$ für die absolutkonvexe Limitierung $\Lambda^{\prime}$ auf $F$ definierend. Nach [3] ist eine $n$-lineare Abbildung $w: E_{1} \times \ldots \times E_{n} \rightarrow F$ genau dann im Nullpunkt $\left(\Lambda_{1} \times \ldots \times \Lambda_{n}, \Lambda^{\prime}\right)$-stetig, wenn $\psi_{1} \ldots \psi_{n} \leq \psi^{\prime w}$ ist.

Für weitere Bezeichnungen wird es auf [3] und [5] verwiesen.

\section{Die projektive absolutkonvexe Tensorproduktlimitierung}

Sei $E$ ein Vektorraum. In $Q(E)$ ist das Supremum einer Familie $\left(q_{\iota}\right)_{\iota} \in I$ von Pseudonormen $q_{\iota} \in Q(E)$ durch

gegeben.

$$
\left(\sup _{\iota \in I} q_{\iota}\right)(x)=\sup _{\iota \in I} q_{\iota}(x)
$$

Es seien $E_{1}, \ldots, E_{n}$ Vektorräume, $t: E_{1} \times \ldots \times E_{n} \rightarrow E_{1} \otimes \ldots \otimes E_{n}$ die kanonische $n$-lineare Abbildung und $q_{v} \in Q\left(E_{v}\right) \quad(v=1, \ldots, n)$ Pseudonorme. Durch

$$
q_{1} \otimes \ldots \otimes q_{n}=\sup _{q^{t} \leq q_{1} \ldots q_{n}} \quad\left(q \in Q\left(E_{1} \otimes \ldots \otimes E_{n}\right)\right)
$$

wird eine Pseudonorm $q_{1} \otimes \ldots \otimes q_{n}$ auf $E_{1} \otimes \ldots \otimes E_{n}$ definiert. Für jedes

$$
z=\sum_{k} x_{1}^{(k)} \otimes \ldots \otimes x_{n}^{(k)} \in E_{1} \otimes \ldots \otimes E_{n}
$$

setzen wir 


$$
p(z)=\inf \sum_{k} q_{1}\left(x_{1}^{(k)}\right) \ldots q_{n}\left(x_{n}^{(k)}\right) \quad(0 \cdot \infty=\infty),
$$

wo das Infimum über alle möglichen Zerlegungen von $z$ der Form (2) genommen wird. Man beweist leicht, dass $p$ eine Pseudonorm ist. Speziell gilt $p^{t} \leq q_{1} \ldots q_{n}$. Sei $q \in Q\left(E_{1} \otimes \ldots \otimes E_{n}\right)$ eine Pseudonorm mit $q^{t} \leq q_{1} \ldots q_{n}$. Dann ist

$$
p(z) \geq \inf \sum_{k} q\left(x_{1}^{(k)} \otimes \ldots \otimes x_{n}^{(k)}\right) \geq \inf q\left(\sum_{k} x_{1}^{(k)} \otimes \ldots \otimes x_{n}^{(k)}\right)=q(z),
$$

d.h. es gilt $p \geq q$. Wir haben somit den ersten Teil des folgenden Satzes bewiesen.

Satz 1. Seien $q_{1}, \ldots, q_{n}$ bzw, $q^{\prime}$ Pseudonormen auf den Vektorräumen $E_{1}, \ldots, E_{n}$ bzw. $F$.

(a) Durch die Gleichungen (1) und (3) wird dieselbe Pseudonorm $p=$ $q_{1} \otimes \ldots \otimes q_{n}$ auf $E_{1} \otimes \ldots \otimes E_{n}$ definiert.

(b) Für jede n-lineare Abbildung $w: E_{1} \times \ldots \times E_{n} \rightarrow F$ mit der assoziierten linearen Abbildung $u: E_{1} \otimes \ldots \otimes E_{n} \rightarrow F, w=u \circ t$, gilt

$$
\left(q^{\prime} \mid q_{1} \ldots q_{n}\right)(w)=\left(q^{\prime} \mid q_{1} \otimes \ldots \otimes q_{n}\right)(u) .
$$

Beweis. (b) Nach Gl. (1) folgt aus $q^{\prime w} \leq q_{1} \ldots q_{n}$ die Beziehung $q^{\prime \prime} \leq q_{1} \otimes \ldots \otimes q_{n}$. Umgekehrt folgt nach Gl. (3) die erste Beziehung aus der zweiten. Nach [4], Satz 8 (a), sind die Relationen $q^{\prime w} \leq q_{1} \ldots q_{n}$ und $q^{\prime u} \leq q_{1} \otimes \ldots \otimes q_{n}$ mit $\left(q^{\prime} \mid q_{1} \ldots q_{n}\right)(w) \leq 1$ bzw. $\left(q^{\prime} / q_{1} \otimes \ldots \otimes q_{n}\right)(u) \leq 1$ äquivalent. Da Pseudonormen positiv homogen und die Abbildung $w \mapsto u$ linear ist, folgt die Behauptung aus der Äquivalenz der Beziehungen $\left(q^{\prime} \mid q_{1} \ldots q_{n}\right)(w) \leq 1$ und $\left(q^{\prime} / q_{1} \otimes \ldots \otimes q_{n}\right)(u) \leq 1$.

Für Teilmengen $M_{v} \subset Q\left(E_{v}\right) \quad(v=1, \ldots, n)$ und Filter $\psi_{v}$ auf $Q\left(E_{v}\right)$ $(v=1, \ldots, n)$ setzen wir $M_{1} \otimes \ldots \otimes M_{n}=\left\{q \in Q\left(E_{1} \otimes \ldots \otimes E_{n}\right) \mid\right.$ $\left.q^{t} \leq q_{1} \ldots q_{n}, q_{v} \in M_{v}, \quad v=1, \ldots, n\right\}$ und

$$
\psi_{1} \otimes \ldots \otimes \psi_{n}=\left[\left\{M_{1} \otimes \ldots \otimes M_{n} \mid M_{v} \in \psi_{v}, \quad v=1, \ldots, n\right\}\right] .
$$

Lemma. (a) Seien $q_{v} \in Q\left(E_{v}\right)$ und $q_{v}^{\prime} \in Q\left(E_{v}\right)(v=1, \ldots, n)$ Pseudonormen mit $q_{v} \leq q_{v}^{\prime}$ für jedes $v=1, \ldots, n$. Dann ist

$$
q_{1} \otimes \ldots \otimes q_{n} \leq q_{1}^{\prime} \otimes \ldots \otimes q_{n}^{\prime} .
$$

(b) Sei für jedes $v=1, \ldots, n M_{v}$ eine saturierte Teilmenge von $Q\left(E_{v}\right)$. Dann ist $M_{1} \otimes \ldots \otimes M_{n}$ saturiert.

(c) Falls eine der Mengen $M_{\nu} \subset Q\left(E_{\nu}\right) \quad(r=1, \ldots, n)$ ausgeglichen ist, so ist $M_{1} \otimes \ldots \otimes M_{n}$ ausgeglichen.

Beweis. Die Eigenschaft (a) folgt unmittelbar aus der Definitionsgleichung (1).

(b) Sei $q^{(u)}(\mu=1, \ldots, m)$ eine endliche Familie von Pseudonormen 
aus $M_{1} \otimes \ldots \otimes M_{n}$. Für jedes $\mu$ gibt es dann Pseudonormen $q_{v}^{(\mu)} \in M^{v}$ $(v=1, \ldots, n)$, so dass $q^{(\mu)} \leq q_{1}^{(u)} \otimes \ldots \otimes q_{n}^{(u)}$ ist. Man erhält mit Hilfe von (a):

$$
\left.\sup _{\mu} q^{(\mu)} \leq \sup _{\mu}\left(q_{1}^{(\mu)} \otimes \ldots \otimes q_{n}^{(\mu)}\right) \leq \sup _{\mu} q_{1}^{(\mu)}\right) \otimes \ldots \otimes\left(\sup _{\mu} q_{n}^{(\mu)}\right) .
$$

Da jedes $M_{v}(v=1, \ldots, n)$ saturiert ist, ist $M_{1} \otimes \ldots \otimes M_{n}$ es folglich auch.

(c) Falls z.B. $M_{1}$ ausgeglichen ist, so gilt für jedes $\lambda>0$ : $\lambda\left(M_{1} \otimes \ldots \otimes M_{n}\right)=\left(\lambda M_{1}\right) \otimes \ldots \otimes M_{n}=M_{1} \otimes \ldots \otimes M_{n}$,

d.h. $M_{1} \otimes \ldots \otimes M_{n}$ ist ausgeglichen.

Sei nun der Filter $\psi_{\nu}$ auf $Q\left(E_{\nu}\right)$ für die absolutkonvexe Vektorraumlimitierung $\Lambda_{v}$ auf $E_{v}$ definierend und saturiert $(v=1, \ldots, n)$. Der Filter $\psi_{1} \otimes \ldots \otimes \psi_{n}$ hat die Eigenschaft $(\mathrm{I})$, denn für jedes $M_{v} \in \psi_{v}$ $(v=1, \ldots, n)$ ist $\left(M_{1} \otimes \ldots \otimes M_{n}\right)^{\lambda}=M_{1}^{\lambda} \otimes M_{2} \otimes \ldots \otimes M_{n}$ für be* liebiges $\lambda \neq 0, \lambda \in \mathbf{K}$. Sei $z$ der Vektor in Gl. (2). Für jedes $x_{u}^{(k)}$ gibt es eine Menge $N_{\mu}^{(k)} \in \psi_{\mu}$, so dass $q\left(x_{\mu}^{(k)}\right)<\infty$ für jedes $q \in N_{\mu}^{(k)}$ ist. Wir setzen $N_{n}=\bigcap_{k} N_{\mu}^{(k)}(\mu=1, \ldots, n)$. Für jedes

$$
q \in N_{1} \otimes \ldots \otimes N_{n}, \quad q^{t} \leq q_{1} \ldots q_{n}, \quad q_{\mu} \in N_{\mu} \quad(\mu=1, \ldots, n),
$$

$\mathrm{i}_{\text {st dann }} q(z) \leq \sum_{k} q_{1}\left(x_{1}^{(k)}\right) \ldots q_{n}\left(x_{n}^{(k)}\right)<\infty$.

Der Filter $\psi_{1} \otimes \ldots \otimes \psi_{n}$ hat also auch die Eigenschaft (II). Er definiert somit eine absolutkonvexe Limitierung auf $E_{1} \otimes \ldots \otimes E_{n}$.

Definition. Die von $\psi_{1} \otimes \ldots \otimes \psi_{n}$ definierte absolutkonvexe Limitierung $\Lambda_{1} \otimes_{k} \ldots \otimes_{k} \Lambda_{n}$ heisse die projektive absolutkonvexe Tensorproduktlimitierung auf $E_{1} \otimes \ldots \otimes E_{n}$ (vgl. [7, 8]).

Wir setzen $E_{1} \otimes_{k} \ldots \otimes_{k} E_{n}=\left(E_{1} \otimes \ldots \otimes E_{n}, A_{1} \otimes_{k} \ldots \otimes_{k} A_{n}\right)$.

Satz 2. (Seien $\left.E_{v}, A_{v}\right)(v=1, \ldots, n)$ und $\left(F, A^{\prime}\right)$ absolutkonvexe Limesvektorräume. Eine n-lineare Abbildung $w: E_{1} \times \ldots \times E_{n} \rightarrow F \quad i$ st genau dann im Nullpunkt stetig, wenn die assoziierte lineare Abbildung $u: E_{1} \otimes_{k} \ldots \otimes_{k} E_{n} \rightarrow F$ stetig ist. Die Limitierung $A_{1} \otimes_{k} \ldots \otimes_{k} A_{n}$ ist die feinste absolutkonvexe Limitierung auf $E_{1} \otimes \ldots \otimes E_{n}$, für die die kanonische Abbildung $t: E_{1} \times \ldots \times E_{n} \rightarrow E_{1} \otimes \ldots \otimes E_{n} \quad i m$ Nullpunkt stetig ist.

Beweis. (A) Sei $\psi_{v}$ für $A_{v}(v=1, \ldots, n)$ definierend und saturiert und sei $\psi^{\prime}$ für $\Lambda^{\prime}$ definierend. Es gilt $\psi_{1} \ldots \psi_{n} \leq\left(\psi_{1} \otimes \ldots \otimes \psi_{n}\right)^{t}$, denn für jedes $M_{v} \in \psi_{v}(v=1, \ldots, n)$ gilt $M_{1} \ldots M_{n} \supset\left(M_{1} \otimes \ldots \otimes M_{n}\right)^{t}$ nach der Definition der Menge $M_{1} \otimes \ldots \otimes M_{n}$. Nach [3], Satz 6 , ist $t$ im Nullpunkt stetig.

(B) Sei $w: E_{1} \times \ldots \times E_{n} \rightarrow F$ im Nullpunkt stetig und sei 


$$
u: E_{1} \otimes \ldots \otimes E_{n} \rightarrow F, w=u \circ t,
$$

die $\mathrm{zu} w$ assoziierte lineare Abbildung. Aus $\psi_{1} \ldots \psi_{n} \leq \psi^{\prime w}$ (nach [3], Satz 6 , ist $w$ genau dann im Nullpunkt stetig, wenn diese Relation erfüllt ist) folgt: Für jedes $M_{v} \in \psi_{v}(v=1, \ldots, n)$ gibt es ein $M^{\prime} \in \psi^{\prime}$, so dass $M_{1} \ldots M_{n} \supset\left(M^{\prime u}\right)^{t}$, gilt. $M^{\prime u}$ ist somit eine Teilmenge von $M_{1} \otimes \ldots \otimes M_{n} . \quad$ Man erhält $\psi_{1} \otimes \ldots \otimes \psi_{n} \leq \psi^{\prime \prime}$, d.h. $u$ ist stetig (siehe [3], Satz 9).

Ist umgekehrt $u: E_{1} \otimes_{k} \ldots \otimes_{k} E_{n} \rightarrow F$ stetig, so ist nach (A) $w=u \circ t$ im Nullpunkt stetig.

(C) Sei $t: E_{1} \times \ldots \times E_{n} \rightarrow\left(E_{1} \otimes \ldots \otimes E_{n}, \Lambda\right)$ in bezug auf die absolutkonvexe Limitierung $\Lambda$ im Nullpunkt stetig. Dann ist nach (B) die Identität $i d: E_{1} \otimes_{k} \ldots \otimes_{k} E_{n} \rightarrow\left(E_{1} \otimes \ldots \otimes E_{n}, A\right)$ stetig, d.h. es gilt $\Lambda_{1} \otimes_{k} \ldots \otimes_{k} \Lambda_{n} \geq \Lambda$.

Nach [3], Satz 7, ist die kanonische Abbildung

$$
t: E_{1} \times \ldots \times E_{n} \rightarrow E_{1} \otimes_{i} \ldots \otimes_{k} E_{n}
$$

überall stetig, falls die absolutkonvexen Räume $E_{1}, \ldots, E_{n}$ ausgeglichen sind.

Kor. 2. Mit denselben Bezeichnungen wie in Satz 2 gilt: Falls die Räume $E_{1}, \ldots, E_{n}$ ausgeglichen sind, so $i$ st $w$ genau dann stetig, wenn $u$ stetig ist. Die ausgeglichene Limitierung $\Lambda_{1} \otimes_{k} \ldots \otimes_{k} \Lambda_{n}$ ist die feinste absolutkonvexe Limitierung auf $E_{1} \otimes \ldots \otimes E_{n}$, für die $t$ stetig ist.

Beweis. Die Limitierung $\Lambda_{1} \otimes_{k} \ldots \otimes_{k} \Lambda_{n}$ ist ausgeglichen nach Lemma 1 (b) und (c) und nach [3], Satz 8.

Satz 3. Sei $q_{v}$ eine Pseudonorm auf dem Vektorraum $E_{v}(v=0, \ldots, n)$ und seien $\quad h: E_{0} \otimes\left(E_{1} \otimes \ldots \otimes E_{n}\right) \rightarrow E_{0} \otimes \ldots \otimes E_{n}$ und $k:\left(E_{0} \otimes \ldots \otimes E_{n-1}\right) \otimes E_{n} \rightarrow E_{0} \otimes \ldots \otimes E_{n}$ die natürlichen Abbildungen. Dann ist

$$
q_{0} \otimes\left(q_{1} \otimes \ldots \otimes q_{n}\right)=\left(q_{0} \otimes \ldots \otimes q_{n}\right)^{h}
$$

und

$$
\left(q_{0} \otimes \ldots \otimes q_{n-1}\right) \otimes q_{n}=\left(q_{0} \otimes \ldots \otimes q_{n}\right)^{k} \text {. }
$$

Beweis. Wir beweisen die erste Gleichung. Die Richtigkeit der zweiten kann in derselben Weise nachgewiesen werden. Wir setzen $p_{0}=$ $q_{0} \otimes \ldots \otimes q_{n}$ und $p_{1}=q_{1} \otimes \ldots \otimes q_{n}$. Für jedes $x_{v} \in E_{v}(v=0, \ldots, n)$ gilt $\left(q_{0} \otimes p_{1}\right)^{h^{-1}}\left(x_{0} \otimes \ldots \otimes x_{n}\right)=q_{0}\left(x_{0}\right) \ldots q_{n}\left(x_{n}\right)$. Man erhält mit Hilfe von Gl. (1): $\left(q_{0} \otimes p_{1}\right)^{h^{-1}} \leq p_{0}$. Seien $t_{1}: E_{1} \times \ldots \times E_{n} \rightarrow E_{1} \otimes \ldots \otimes E_{n}$ und $t_{2}: E_{0} \times\left(E_{1} \otimes \ldots \otimes E_{n}\right) \rightarrow E_{0} \otimes\left(E_{1} \otimes \ldots \otimes E_{n}\right)$ die kanonischen Abbildungen. Die Abbildung $\bar{x}_{0}: E_{1} \otimes \ldots \otimes E_{n} \rightarrow E_{0} \otimes\left(E_{1} \otimes \ldots \otimes E_{n}\right)$, die durch $\bar{x}_{0}\left(x_{1} \otimes \ldots \otimes x_{n}\right)=x_{0} \otimes\left(x_{1} \otimes \ldots \otimes x_{n}\right)$ definiert ist, ist für 
jedes $x_{0} \in E_{0}$ linear. Offenbar gilt $p_{0}^{h_{0} \bar{x}_{0} \circ t_{1}} \leq q_{0}\left(x_{0}\right) q_{1} \ldots q_{n}$ für jedes $x_{0} \in E_{0}$. Hieraus folgt $p_{0}^{h_{0} \bar{x}_{0}} \leq q_{0}\left(x_{0}\right) p_{1}$ und ferner $p_{0}^{h_{0} t_{2}} \leq q_{0} p_{1}$. Wir erhalten nun schliesslich die Ungleichung $p_{0}^{h} \leq q_{0} \otimes p_{1}$. Es gilt somit $p_{0}^{h}=q_{0} \otimes p_{1}$ und die erste Gleichung ist damit bewiesen.

Aus Satz 3 folgt unmittelbar:

Satz 4. Seien $E_{v}(v=0, \ldots, n)$ absolutkonvexe Limesvektorräume. Dann sind die natürlichen Abbildungen

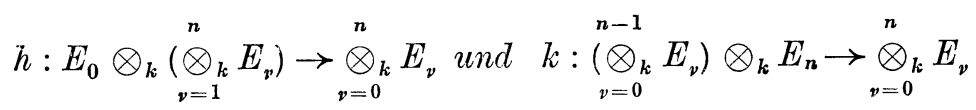

lineare Homöomorphismen.

Offensichtlich gilt auch

Satz 5. Für je zwei absolutkonvexe Limesvektorräume $E$ und $F$ sind $E \otimes_{k} F$ und $F \otimes_{k} E$ in kanonischer Weise homöomorph.

Satz 6. Seien $u_{i}: E_{i} \rightarrow F_{i} \quad(i=1,2)$ stetige lineare Abbildungen zwischen den absolutkonvexen Limesvektorräumen $E_{i}$ und $F_{i}(i=1,2)$. Dann ist ihr Kronecker-Produkt $u_{1} \otimes u_{2}: E_{1} \otimes_{k} E_{2} \rightarrow F_{1} \otimes_{k} F_{2}$ stetig. $u_{1} \otimes u_{2}$ ist ein linearer Homöomorphismus, wenn dies für $u_{1}$ und $u_{2}$ gilt.

Beweis. $u_{1} \otimes u_{2}$ ist die $\mathrm{zu}$ der im Nullpunkt stetigen bilinearen Abbildung $t \circ\left(u_{1} \times u_{2}\right)$ assoziierte lineare Abbildung und ist folglich stetig $\left(t\right.$ bezeichnet die kanonische Abbildung $F_{1} \times F_{2} \rightarrow F_{1} \otimes F_{2}$ ). Falls $v_{i}$ eine stetige Inverse $\mathrm{zu} \quad u_{i}$ ist $(i=1,2)$, so ist $v_{1} \otimes v_{2}$ eine stetige Inverse $\mathrm{zu} \quad u_{1} \otimes u_{2}$.

Ferner gilt

Satz 7. Jeder ausgeglichene, absolutkonvexe Limesvektorraum $E$ ist in kanonischer Weise zu $\mathbf{K} \otimes_{k} E$ homöomorph.

Beweis. Der kanonische Isomorphismus $s: \mathbf{K} \otimes E \rightarrow E, s: \lambda \otimes x \mapsto \lambda x$, ist stetig, da die Skalarmultiplikation stetig ist.

Der saturierte Filter $\psi_{0}=\left[\left\{\Gamma_{0}\right\}\right], \quad \Gamma_{0}=\left\{q \in Q(\mathbf{K}) \mid q \leq \lambda p_{0}, \lambda>0\right\}$, wo $p_{0}$ die natürliche Norm auf $\mathbf{K}$ bezeichnet, ist für die natürliche Topologie auf $\mathbf{K}$ definierend. Sei $\psi$ für die absolutkonvexe und ausgeglichene Limitierung auf $E$ definierend und saturiert (vgl. [3], Satz 8). Für jedes ausgeglichene, saturierte $M \in \psi$ gilt $M \supset\left(\Gamma_{0} \otimes M\right)^{s^{-1}}$ denn für jedes $q \in \Gamma_{0} \otimes M$ mit $q^{t} \leq \lambda p_{0} q, \lambda>0, q \in M$, ist $q^{s^{-1}}(x)=q^{t}(1, x) \leq \lambda q(x)$ für jedes $x \in E$. Es gilt somit $\psi \leq\left(\psi_{0} \otimes \psi\right)^{s^{-1}}$, d.h. $s^{-1}$ ist stetig.

Seien $E_{1}, \ldots, E_{n}, F$ absolutkonvexe Limesvektorräume und sei ${ }^{\circ} \mathscr{L}\left(E_{1}, \ldots, E_{n} ; F\right)$ die Menge der im Nullpunkt stetigen, $n$-linearen Abbildungen $w: E_{1} \times \ldots \times E_{n} \rightarrow F$. Für die gröbste ausgeglichene, absolutkonvexe Limitierung $\Lambda_{p}$ auf ${ }^{\circ} \mathscr{L}\left(E_{1}, \ldots, E_{n} ; F\right)$, für die die Evaluation $\omega:{ }^{\circ} \mathscr{L}\left(E_{1}, \ldots, E_{n} ; F\right) \times E_{1} \times \ldots \times E_{n} \rightarrow F$ im Nullpunkt stetig ist, gilt 
nach [4]: Für jeden ausgeglichenen, absolutkonvexen Raum $Z$ und jedes lineare $f: Z \rightarrow\left({ }^{\circ} \mathscr{L}\left(E_{1}, \ldots, E_{n} ; F\right), A_{p}\right)={ }^{\circ} \mathscr{L}_{p}\left(E_{1}, \ldots, E_{n} ; F\right)$ ist $f$ genau dann stetig, wenn die assoziierte $(n+1)$-lineare Abbildung $\tilde{f}: Z \times E_{1} \times \ldots \times E_{n} \rightarrow F$ im Nullpunkt stetig ist. Diese Eigenschaft der Limitierung $\Lambda_{p}$ wird im Beweis des nächsten Satzes benutzt.

Im folgenden bezeichnet $\hat{w}: E_{1} \otimes \ldots \otimes E_{n} \rightarrow F$ die zu der $n$-linearen Abbildung $w: E_{1} \times \ldots \times E_{n} \rightarrow F$ assoziierte lineare Abbildung. $\mathscr{L}\left(E_{1}, \ldots, E_{n} ; F\right)$ sei die Menge aller stetigen $w \in{ }^{\circ} \mathscr{L}\left(E_{1}, \ldots, E_{n} ; F\right)$.

Satz 8. Seien $E_{1}, \ldots, E_{n}$ und $F$ absolutkonvexe Limesvektorräume Dann ist die durch $\xi: w \mapsto \hat{w}$, definierte natürliche Abbildung

$$
\xi:{ }^{\circ} \mathscr{L}_{p}\left(E_{1}, \ldots, E_{n} ; F\right) \rightarrow \mathscr{L}_{p}\left(E_{1} \otimes_{k} \ldots \otimes_{k} E_{n} ; F\right)
$$

ein linearer Homöomorphismus.

Beweis. Sei

$$
\begin{gathered}
h:{ }^{\circ} \mathscr{L}_{p}\left(E_{1}, \ldots, E_{n} ; F\right) \otimes_{k}\left(E_{1} \otimes_{k} \ldots \otimes_{k} E_{n}\right) \rightarrow \\
{ }^{\circ} \mathscr{L}_{p}\left(E_{1}, \ldots, E_{n} ; F\right) \otimes_{k} E_{1} \otimes_{k} \ldots \otimes_{k} E_{n}
\end{gathered}
$$

der kanonische Homöomorphismus. Für die zu der bilinearen Abbildung $\alpha(\xi):{ }^{\circ} \mathscr{L}\left(E_{1}, \ldots, E_{n} ; F\right) \times\left(E_{1} \otimes \ldots \otimes E_{n}\right) \rightarrow F \quad$ assoziierte Abbildung $\widehat{\alpha(\xi)}:{ }^{\circ} \mathscr{L}\left(E_{1}, \ldots, E_{n} ; F\right) \otimes\left(E_{1} \otimes \ldots \otimes E_{n}\right) \rightarrow F$ gilt $\widehat{\alpha(\xi)}=\hat{\omega} \circ h$. Da die Evaluation $\omega:{ }^{\circ} \mathscr{L}_{p}\left(E_{1}, \ldots, E_{n} ; F\right) \times E_{1} \times \ldots \times E_{n} \rightarrow F$ im Nullpunkt stetig ist, ist die Abbildung $\xi$ stetig. Ähnlich folgt, dass $\xi^{-1}$ stetig ist. Es gilt nämlich $\widehat{\alpha\left(\xi^{-1}\right)}=\hat{\omega}_{1} \circ h_{1}^{-1}$, wo $\omega_{1}$ die Evaluation

und

$$
\mathscr{L}\left(E_{1} \otimes_{k} \ldots \otimes_{k} E_{n} ; F\right) \times\left(E_{1} \otimes \ldots \otimes E_{n}\right) \rightarrow F
$$

$$
\begin{gathered}
h_{1}: \mathscr{L}_{p}\left(E_{1} \otimes_{k} \ldots \otimes_{k} E_{n} ; F\right) \otimes_{k}\left(E_{1} \otimes_{k} \ldots \otimes_{k} E_{n}\right) \rightarrow \\
\mathscr{L}_{p}\left(E_{1} \otimes_{k} \ldots \otimes_{k} E_{n} ; F\right) \otimes_{k} E_{1} \otimes_{k} \ldots \otimes_{k} E_{n}
\end{gathered}
$$

der kanonische Homöomorphismus ist.

Bemerkung. Der für $\Lambda_{p}$ definierende Filter wurde in [4] durch Pseudonormen der Form $q^{\prime} / q_{1} \ldots q_{n}$ charakterisiert. Offenbar kann man den obigen Satz auch mit Hilfe von Satz 1 (b) beweisen.

Sei $\Lambda_{c}$ die Limitierung der stetigen Konvergenz (siehe [2]). Genau wie oben beweist man Satz 9. Zu beachten ist, dass $\Lambda_{c}$ nur auf $\mathscr{L}\left(E_{1}, \ldots, E_{n} ; F\right)$ definiert ist.

Satz 9. Seien $E_{1}, \ldots, E_{n}$ ausgeglichene, absolutkonvexe Limesvektorräume und $F$ ein absolutkonvexer Limesvektorraum. Dann ist die natürliche Abbildung $\xi: \mathscr{L}\left(E_{1}, \ldots, E_{n} ; F\right) \rightarrow \mathscr{L}\left(E_{1} \otimes_{k} \ldots \otimes_{k} E_{n} ; F\right)$ ein linearer $\left(\Lambda_{c}, \Lambda_{c}\right)$-Homöomorphismus. 
Satz 10. Seien $E_{i}$ und $F_{i}(i=1,2)$ absolutkonvexe Limesvektorräume. Dann ist die durch $(u, v) \mapsto u \otimes v$ definierte bilineare Abbildung $\eta: \mathscr{L}\left(E_{1} ; F_{1}\right) \times \mathscr{L}\left(E_{2} ; F_{2}\right) \rightarrow \mathscr{L}\left(E_{1} \otimes_{k} E_{2} ; F_{1} \otimes_{k} F_{2}\right) \quad\left(\Lambda_{p} \times \Lambda_{p}, \Lambda_{p}\right)$-stetig und im Nullpunkt $\left(\Lambda_{c} \times \Lambda_{c}, \Lambda_{c}\right)$-stetig.

Beweis. Das Diagramm

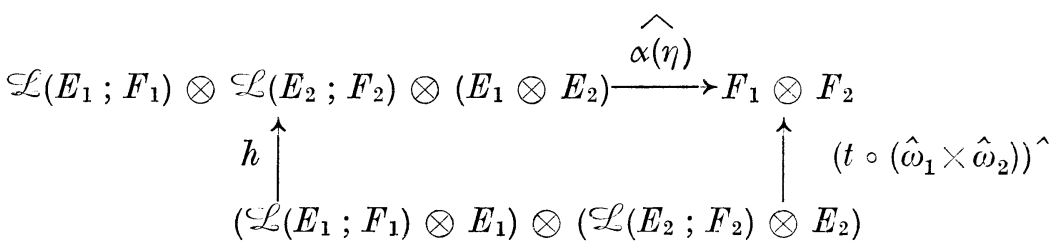

wo $h$ die natürliche Abbildung, $\omega_{i}: \mathscr{L}\left(E_{i} ; F_{i}\right) \times E_{i} \rightarrow F_{i} \quad(i==1,2)$ die Evaluation und $t: F_{1} \times F_{2} \rightarrow F_{1} \otimes F_{2}$ die kanonische Abbildung ist, ist kommutativ. Da $\omega_{1}$ und $\omega_{2}$ bezüglich $\Lambda_{p}$ und $\Lambda_{c}$ stetig sind (siehe [4] und [2]), folgt die Behauptung hieraus. Die Limitierung $\Lambda_{c}$ ist nämlich absolutkonvex nach [4], Satz 6 .

Sei $(E, A)$ ein absolutkonvexer Limesvektorraum und sei $L$ ein linearer Unterraum von $E$. Wir bezeichnen mit $x \mapsto \bar{x}$ die kanonische Abbildung aus $E$ nach $E / L$. Jeder Pseudonorm $q$ auf $E$ ist eine Pseudonorm $\bar{q}$ auf $E / L, \bar{q}(\bar{x})=\inf \{q(y) \mid y \in \bar{x}\}$, zugeordnet. Falls $\psi$ die Limitierung $A$ definiert, so ist $\bar{\psi}=[\{\bar{M} \mid M \in \psi\}]$ für die Quotientenlimitierung auf $E / L$ definierend ([3], Satz 4). Dabei ist $\bar{M}=\{\bar{q} q \in M\}$.

Satz 11. Es sei $E$ bzw. $F$ ein absolutkonvexer Limesvektorraum mit dem Unterraum $G$ bzw. H. Dann ist die kanonische Abbildung $(E / G) \otimes_{k}(F / H) \rightarrow\left(E \otimes_{k} F\right) / \Gamma(G, H)$, wo $\Gamma(G, H)$ den von der Menge $\{x \otimes y \mid x \in G$ oder $y \in H\}$ erzeugten Unterraum bezeichnet, ein linearer Homöomorphismus.

Beweis. Sei $u: \bar{x} \otimes \bar{y} \rightarrow \overline{x \otimes y}$ der kanonische, lineare Isomorphismus. Es genügt zu zeigen, dass für beliebige Pseudonorme $q_{1}$ und $q_{2}$ auf $E$ bzw. $F \quad \bar{q}_{1} \otimes \bar{q}_{2}=\left(\overline{q_{1} \otimes q_{2}}\right)^{u}$ ist. Für jedes $x \in E$ und jedes $y \in F$ ist $\left(\overline{q_{1} \otimes q_{2}}\right)(\overline{x \otimes y}) \leq q_{1}(x) q_{2}(y)$. Folglich gilt $\quad\left(\overline{q_{1} \otimes q_{2}}\right)(\overline{x \otimes y}) \leq \bar{q}_{1}(\bar{x}) \bar{q}_{2}(\bar{y})$, woraus nach Gl. (1) die Ungleichung $\left(\overline{q_{1} \otimes q_{2}}\right)^{u} \leq \bar{q}_{1} \otimes \bar{q}_{2}$ folgt. Sei $a \in(E / G) \otimes(F / H)$ und $z \in u(a)$ beliebig. Für jede Zerlegung $z=\sum x^{(k)} \otimes y^{(k)}$ von $z$ ist $\sum \bar{x}^{(k)} \otimes \bar{y}^{(k)}$ eine Zerlegung ron $a$. Man erhält mit Hilfe von (3):

$$
\left(\bar{q}_{1} \otimes \bar{q}_{2}\right)(a) \leq \inf \sum_{k} \bar{q}_{1}\left(\bar{x}^{(k)}\right) \bar{q}_{2}\left(\bar{y}^{(k)}\right) \leq \inf \sum_{k} q_{1}\left(x^{(k)}\right) q_{2}\left(y^{(k)}\right)=\left(q_{1} \otimes q_{2}\right)(z),
$$

wo das Infimum über alle möglichen Zerlegungen von $z$ genommen wird. Da dieses für jedes $z \in u(a)$ und jedes $a$ gilt, ist $\left.\bar{q}_{1} \otimes \bar{q}_{2} \leq \overline{\left(q_{1} \otimes q_{2}\right.}\right)^{u}$. Es gilt somit $\bar{q}_{1} \otimes \bar{q}_{2}=\left(\overline{q_{1} \otimes q_{2}}\right)^{u}$. 
Sei $(E, A)$ ein ausgeglichener, absolutkonvexer Limesvektorraum und sei der saturierte Filter $\psi$ für $\Lambda$ definierend. Durch

$$
q_{M}(x)=\left\{\begin{array}{l}
0 \text { falls } q(x)<\infty \text { für jedes } q \in M \text { ist } \\
\infty \text { falls es ein } q \in M \text { mit } q(x)=\infty \text { gibt }
\end{array}\right.
$$

wird für jede Teilmenge $M$ von $Q(E)$ eine Pseudonorm $q_{M}$ auf $E$ definiert. Die Einschränkungen auf $L_{M}=q_{M}^{-1}(0)$ der Pseudonormen in $M$ sind Seminormen und definieren eine lokalkonvexe Vektorraumtopologie $\tau_{M}$ auf $L_{M}$. Sei $\chi$ eine Filterbasis von $\psi$ aus lauter ausgeglichenen, saturierten Mengen. Für je zwei $M, N \in \chi$ mit $M \supset N$ sei $i_{N M}: L_{M} \rightarrow L_{N}$ die Inklusion. Diese ist offensichtlich $\left(\tau_{M}, \tau_{N}\right)$-stetig. Falls für jedes $M \in \chi$ die Beziehung $q_{M} \in M$ erfüllt ist, so ist $(E, A)$ induktiver Limes (in der Kategorie der Limesvektorräume) der gerichteten Familie $\left(L_{M}, \tau_{M}\right)_{M \in \varkappa}$ von lokalkonvexen Räumen. Für jedes $M \in \chi$ ist nämlich dann $i_{M}\left(\varkappa_{M}\right)=$ $\mathcal{F}_{M}$, wobei ${ }^{c} \ell_{M}$ der Nullumgebungsfilter im $\operatorname{Raum}\left(L_{M}, \tau_{M}\right)$ und $i_{M}: L_{M} \rightarrow E$ die Inklusion bezeichnet (siehe [8], Satz 1.2.2). $(E, A)$ ist also ein Marinescu-Raum.

Wir setzen nun umgekehrt voraus, dass $(E, A)$ ein Marinescu-Raum ist. Dieser Raum ist dann bezüglich stetiger Inklusionen $i_{\varkappa i}: E_{\iota} \rightarrow E_{\varkappa}, \iota \leq \varkappa$, induktiver Limes einer durch $\leq$ gerichteten Familie $\left(E_{\imath}, \tau_{\imath}\right)_{\imath} \in I$ von lokalkonvexen Räumen. Sei für jedes $\iota \in I \quad M$, die Menge der $\tau$-stetigen Seminormen auf $E_{\imath}$. Jedes $q \in M$ kann als Pseudonorm auf ganz $E$ fortgesetzt werden: Wir setzen $q^{\prime}(x)=q(x)$ falls $x \in E$, und $q^{\prime}(x)=\infty$ falls $x \notin E_{\imath}$ ist. Ferner setzen wir $M_{\imath}^{\prime}=\left\{q^{\prime} \mid q \in M\right\}$ für jedes $\iota \in I$. Die Filterbasis $\left\{M_{\imath}^{\prime} \mid \iota \in I\right\}$ erzeugt einen saturierten Filter auf $Q(E)$, der $\Lambda$ definiert. Für jedes $\iota \in I$ ist $M_{\iota}^{\prime}$ ausgeglichen und es gilt $q_{M_{\iota}^{\prime}} \in M_{\imath}^{\prime}$.

Wir fassen zusammen:

Satz 12. Ein ausgeglichener, absolutkonvexer Limesvektorraum $(E, \Lambda)$ mit dem saturierten, definierenden Filter $\psi$ ist genau dann ein MarinescuRaum, wenn die Beziehung $q_{M} \in M$ für jedes $M$ in einer Filterbasis $\chi$ von $\psi$ aus lauter ausgeglichenen, saturierten Mengen gilt. Dabei ist für jedes $M \in \chi$ die Pseudonorm $q_{M}$ durch (4) definiert.

Dieser Satz kann auch aus einer von K. Kutzler in [11] gegebenen internen Charakterisierung der Marinescu-Räume hergeleitet werden.

Satz 13. Für je zwei Marinescu-Räume $E$ und $F$ ist $E \otimes_{k} F$ ein Marinescu-Raum.

Beweis. Der saturierte Filter $\psi_{1}$ (bzw. $\psi_{2}$ ) sei für die Limitierung auf $E$ (bzw. $F$ ) definierend. $\psi_{1}$ (bzw. $\psi_{2}$ ) besitzt nach Satz 12 eine Basis $\chi_{1}$ (bzw. $\chi_{2}$ ) aus ausgeglichenen, saturierten Mengen derart, dass für jedes $M \in \chi_{1}$ (bzw. $M \in \chi_{2}$ ) die Beziehung $q_{M} \in M$ erfüllt ist. Für beliebiges 
$M_{i} \in \chi_{i} \quad(i=1,2) \quad$ ist $\quad q_{M_{1} \otimes M_{2}}(x \otimes y) \leq q_{M_{1}}(x) q_{M_{2}}(y)$ für jedes $x \in E$, $y \in F$, denn falls $q_{1}(x)$ und $q_{1}(y)$ für jedes $q_{i} \in M_{i}(i=1,2)$ endlich sind, so gilt dasselbe für $q(x \otimes y)$ für jedes $q \in M_{1} \otimes M_{2}$. Man erhält $q_{M_{1} \otimes M_{2}} \leq q_{M_{1}} \otimes q_{M_{2}} . \quad$ Da $\left\{M_{1} \otimes M_{2} \mid M_{i} \in \chi_{i}, \quad i=1,2\right\}$ eine Basis von $\psi_{1} \otimes \psi_{2}$ aus ausgeglichenen, saturierten Mengen ist (Lemma 1), so ist die von $\psi_{1} \otimes \psi_{2}$ definierte Limitierung eine Marinescu-Limitierung (Satz 12). Für jedes $M_{i} \in \chi_{i} \quad(i=1,2)$ ist ja $q_{M_{1} \otimes M_{2}} \in M_{1} \otimes M_{2}$.

Bemerkung. Die projektive, absolutkonvexe Tensorproduktlimitierung auf $E \otimes F$ ist offenbar die feinste Marinescu-Limitierung, für die die kanonische Abbildung $t: E \times F \rightarrow E \otimes F$ stetig ist, falls $E$ und $F$ Marinescu-Räume sind. Sie fällt somit in diesem Fall mit einer von $H$. Jarchow eingeführten Tensorproduktlimitierung zusammen ([8], Satz 2.3.9). Falls $E$ und $F$ lokalkonvexe, topologische Vektorräume sind, so ist die projektive, absolutkonvexe Tensorproduktlimitierung auf $E \otimes F$ mit der von A. Grotendieck in [7] eingeführten projektiven Tensorprodukttopologie identisch (siehe [8], Seite 152).

Bemerkung. Seien $\left(E, \Lambda_{1}\right)$ und $\left(F, \Lambda_{2}\right)$ absolutkonvexe Limesvektorräume. Mit Hilfe einer Beweisführung in [8] (Seite 153) beweist man leicht, dass mit $\Lambda_{1} \otimes_{k} \Lambda_{2}$ auch $\Lambda_{1}$ und $\Lambda_{2}$ separiert sind. Umgekehrt folgt aus [8], Satz 2.3.11, und aus Satz 13, dass $\Lambda_{1} \otimes_{k} \Lambda_{2}$ separiert ist, falls $\Lambda_{1}$ und $\Lambda_{2}$ separierte Marinescu-Limitierungen sind.

\section{Direkte Summen und Tensoralgebren}

Sei $\left(E_{\imath}\right)_{\iota \in I}$ eine nichtleere Familie von Vektorräumen und $\left(q_{\iota}\right)_{\iota} \in I$ eine Familie von Pseudonormen $q_{\imath} \in Q\left(E_{\imath}\right)$. Durch

$$
\left.\underset{\iota \in I}{\oplus} q_{\iota}=\sup _{q^{i_{\iota}} \leq q_{\iota} ; \iota \in I} q \in \underset{\iota \in I}{\oplus} E_{\iota}\right)
$$

wird eine Pseudonorm $\oplus_{\iota I} q_{\iota}$ auf $\oplus_{\in I} E_{\iota}$ definiert. Hierbei bezeichnet $i_{\imath}: E_{\iota} \rightarrow \oplus E_{\varkappa}$ für jedes $\iota \in I$ die natürliche Injektion. Die Pseudonorm $\oplus q_{\iota}$ ist gleich der Pseudonorm $p$, die an der Stelle $z=\sum_{\iota \in I} x_{\iota} \in \underset{\iota \in I}{\oplus} E_{\iota}$ $\left(x_{\imath} \in E_{\imath}, x_{\imath} \neq 0\right.$ nur für endlich viele $\left.\iota \in I\right)$ den Wert

$$
p(z)=\sum_{\imath \in I} q_{\imath}\left(x_{\imath}\right)
$$

annimmt.

Für jeden Vektorraum $E$ sei $q_{\infty}$ das grösste Element im Verband $Q(E) . I^{*}$ bezeichne die Menge der endlichen Teilmengen von $I$. Für jedes $J \in I^{*}$ und jede Familie $\left(M_{\imath}\right)_{\iota \in J}$ von Teilmengen $M_{\imath} \subset Q\left(E_{\imath}\right)$, setzen wir 
$\underset{\iota \in J}{\oplus} M_{\iota}=\left\{q \in Q(\oplus E) \mid q \leq \oplus q_{\iota}, q_{\iota} \in M_{\iota}\right.$ für $\iota \in J$ und $q_{\iota}=q_{\infty}$ für $\left.\iota \notin J\right\}$.

Sei nun $\left(\left(E_{\imath}, \Lambda_{\imath}\right)\right)_{\iota \in I}$ eine Familie von absolutkonvexen Limesvektorräumen und sei der saturierte Filter $\psi_{\iota}$ für jedes $\iota \in I$ für $\Lambda$, definierend. Wir setzen

$$
\left.\underset{\iota \in I}{\oplus} \psi_{\iota}=\left[\underset{\imath \in I}{\underset{f}{\oplus}} M_{\imath} \mid M \in \psi_{\imath} \text { für jedes } \iota \in J, J \in I^{*}\right\}\right] .
$$

Dieser Filter ist saturiert und genügt (I) und (II). Er definiert somit eine absolutkonvexe Limitierung $\underset{\iota \in I}{\oplus} \Lambda_{\imath}$ auf $\underset{\iota \in I}{\oplus} E_{\imath}$.

Satz 14. Eine lineare Abbildung $f: \oplus E_{*} \rightarrow F$ nach einem absolutkonvexen Limesvektorraum $\left(F, \Lambda^{\prime}\right)$ ist genau dann $\left(\oplus \Lambda_{\varkappa}, \Lambda^{\prime}\right)$-stetig, wenn für jedes $\iota \in I$ die Abbildung $f \circ i_{\iota}\left(\Lambda_{\iota}, \Lambda^{\prime}\right)$-stetig ist. Für jedes $\iota \in I$ ist $i_{\iota}: E_{\iota} \rightarrow \oplus E_{\varkappa}$ eine Einbettung.

Beweis. Für jedes $\iota \in I$ ist $\psi_{\iota}=\left(\oplus \psi_{\varkappa}\right)^{i} \iota$, d.h. $i$, ist eine Einbettung ([3], Satz 11). Ist $f\left(\oplus \Lambda_{\varkappa}, A^{\prime}\right)$-stetig, so ist folglich $f \circ i_{i}\left(\Lambda_{\imath}, A^{\prime}\right)$-stetig für jedes $\iota \in I$.

Wir nehmen nun umgekehrt an, dass $f \circ i$, für jedes $\iota \in I \quad\left(\Lambda_{\imath}, \Lambda^{\prime}\right)$ stetig ist. Sei der Filter $\psi^{\prime}$ für $\Lambda^{\prime}$ definierend. Dann ist $\psi_{\imath} \leq \psi^{\prime f c i}$, d.h. für jedes $\iota \in I$ und jedes $M_{\imath} \in \psi_{\imath}$ gibt es ein $H_{\imath}^{\prime} \in \psi^{\prime}$ derart, dass $M_{\iota} \supset\left(M_{\imath}^{\prime}\right)^{i_{\iota}}$ gilt. Für jedes $J \in I^{*}$ setzen wir $M_{J}^{\prime}=\cap\left\{M_{\imath}^{\prime} \iota_{\iota} \in J\right\}$. Nach der Definition der Menge $\underset{\iota \in J}{\oplus} M_{\iota}$ ist diese Menge Obermenge zu $M_{J}^{\prime}$. Man erhält: $\oplus \psi_{\iota} \leq \psi^{\prime} f$, d.h. $f$ ist stetig.

Bemerkung. Aus Satz 14 folgt, dass $\oplus A_{\varkappa}$ die feinste absolutkonvexe Limitierung auf $\oplus E_{\varkappa}$ ist, für die $i_{\imath}: E_{\iota} \rightarrow \oplus E_{\%}$ für jedes $\iota \in I$ stetig ist. H. R. Fischer hat in [5] eine Limitierung auf $\oplus E_{*}$ als induktiver Limes der Unterräume $\left(\left(\oplus_{\epsilon \in J} E_{\iota}, \prod_{\epsilon \in J} A_{\iota}\right)\right)_{J \in I^{*}}$ bezüglich der natürlichen Inklusionen definiert. Diese Limitierung ist nach [8] die feinste Vektorraumlimitierung, für die alle $i$, stetig sind. Da Produkte und induktive Limites (in der Kategorie der Limesvektorräume) von absolutkonvexen Räumen absolutkonvex sind (die in [12] hierfür gegebene Beweise sind für den reellen Fall durchgeführt aber können unmittelbar rerallgemeinert werden), so ist folglich $\oplus \Lambda_{\varkappa}$ gleich der von Fischer definierten Limitierung. Für weitere Eigenschaften der Limitierung $\oplus \Lambda_{\varkappa}$ verweisen wir deswegen auf $[8]$.

Satz 15. Es seien $\left(E_{\imath}\right)_{\imath \in I}$ und $\left(F_{x}\right)_{x \in K}$ Vektorräume $(I \neq \phi, K \neq \phi)$,

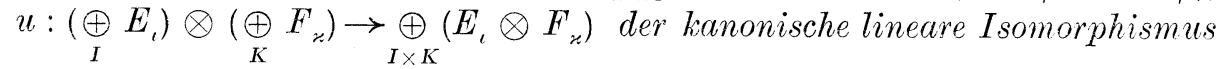
und $\left(q_{\imath}\right)_{t \in I}$ und $\left(p_{x_{x}}\right)_{x \in K}$ Familien von Pseudonormen mit $q_{1} \in Q\left(E_{\imath}\right)$ bzu. $p_{\varkappa} \in Q\left(F_{\varkappa}\right)$ für jedes $\iota \in I$ bzw. $x \in K$. Dann ist 


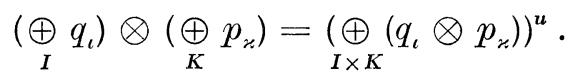

Beweis. Sei $i_{\iota \varkappa}: E_{\iota} \otimes F_{x} \rightarrow \oplus\left(E_{\lambda}^{\prime} \otimes F_{\mu}\right)$ für jedes $\iota \in I, \quad x \in K$ die natürliche Injektion. Für beliebiges $\iota \in I, x \in K$ und für jedes $x_{\imath} \in E_{\imath}$, $y_{\varkappa} \in F_{\varkappa}$ ist $\left(\left(\oplus q_{\lambda}\right) \otimes\left(\oplus p_{\mu}\right)\right)^{u^{-1} \iota_{\imath} i_{\varkappa}}\left(x_{\iota} \otimes y_{\varkappa}\right) \leq q_{\iota}\left(x_{\iota}\right) p_{\varkappa}\left(y_{\varkappa}\right)$. Hieraus erhält man mit Hilfe von (1) und (5) die Ungleichung

$$
\left(\left(\oplus q_{\lambda}\right) \otimes\left(\oplus p_{\mu}\right)\right)^{u^{-1}} \leq \oplus\left(q_{\lambda} \otimes p_{\mu}\right) .
$$

Andererseits gilt für jedes $\sum x_{\iota} \in \oplus E_{\iota}, x_{\iota} \in E_{\iota}{ }^{,}$, und jedes $\sum y_{\varkappa} \in \oplus F_{\psi}$, $y_{\varkappa} \in F_{\varkappa}$, die Beziehung

$$
\begin{gathered}
\underset{I \times K}{\left.\left(\oplus q_{\iota} \otimes p_{\varkappa}\right)\right)^{u}}\left(\left(\sum_{I} x_{\iota}\right) \otimes\left(\sum_{K} y_{\varkappa}\right)\right) \leq \underset{I \times K}{\oplus} q_{\iota}\left(x_{\iota}\right) p_{\varkappa}\left(y_{\varkappa}\right)= \\
=\left(\underset{I}{\oplus} q_{\iota}\right)\left(\sum_{I} x_{\imath}\right)\left(\underset{K}{\oplus} p_{\varkappa}\right)\left(\sum_{K} y_{\varkappa}\right),
\end{gathered}
$$

woraus mit Hilfe von (1) die umgekehrte Ungleichung folgt. Satz 15 ist damit bewiesen.

Aus Satz 15 folgt:

Satz 16. Für beliebige nichtleere Familien $\left(\left(E_{\imath}, \Lambda_{\imath}\right)\right)_{\iota \in I}$ und $\left(\left(F_{\varkappa}, \Lambda_{\varkappa}^{\prime}\right)\right)_{\varkappa \in K}$ von absolutkonvexen Limesvektorräumen ist die kanonische Abbildung $\left.u:\left(\underset{I}{\oplus} E_{\imath}\right) \otimes \underset{K}{\left(\oplus_{\kappa}\right.} F_{z}\right) \rightarrow \underset{I \times K}{\oplus}\left(E_{\imath} \otimes F_{r}\right) \quad$ ein $\quad$ linearer $\quad\left(\left(\oplus \Lambda_{\imath}\right) \otimes_{k}\left(\oplus A_{r}^{\prime}\right)\right.$, $\left.\oplus\left(\Lambda_{\iota} \otimes_{k} \Lambda_{\varkappa}^{\prime}\right)\right)$-Homöomorphismus.

Bemerkung. Sei $\left(\left(E_{\imath}, A_{\imath}\right)\right)_{\iota \in I}$ eine Familie von absolutkonvexen Räumen und sei der saturierte Filter $\psi_{\iota}$ für jedes $\iota \in I$ für $\Lambda$, definierend. Wir setzen $\oplus_{t} \psi_{\imath}=\left[\left\{\oplus M_{\imath} \mid M_{\imath} \in \psi_{\imath}\right.\right.$ für jedes $\left.\left.\iota \in I\right\}\right]$, wobei $\oplus M_{\imath}$ die Menge $\left\{q \in Q\left(\oplus E_{\imath}\right) \mid q \leq \oplus q_{\imath}, q, \in M\right.$, für jedes $\left.\iota \in I\right\}$ bezeichnet. $\oplus_{t} \psi_{\imath}$ definiert eine Limitierung $\oplus_{t} \Lambda_{\imath}$ auf $\oplus E_{t}$, die folgende Eigenschaft hat: Für jeden lokalkonvexen (topologischen) $\operatorname{Raum}(Z, \tau)$ und jedes lineare $f: \oplus E \rightarrow Z$ ist $f$ genau damn $\left(\oplus_{t} A_{\imath}, \tau\right)$-stetig, wenn für jedes $\iota \in I$ die Abbildung $f \circ i_{\iota}\left(\Lambda_{\imath}, \tau\right)$-stetig ist. Falls die Räume $\left(\left(E,, A_{\imath}\right)\right)_{\iota} \in I$ lokalkonvex sind, so ist $\left(\oplus E_{\imath}, \oplus_{t} \Lambda_{\imath}\right)$ offensichtlich gleich der üblichen topologischen, direkten Summe dieser Räume. Aus Satz 15 folgt, dass die Bildung der direkten Summe $\oplus_{t} \Lambda_{\iota}$ mit der Bildung der projektiven, absolutkonvexen Tensorproduktlimitierung verträglich ist, d.h. dass die kanonische Abbildung $u$ in Satz 16 auch ein $\left(\left(\oplus_{t} A_{\imath}\right) \otimes_{k}\left(\oplus_{t} A_{r}^{\prime}\right)\right.$, $\left.\oplus_{t}\left(\Lambda_{1} \otimes_{k} \Lambda_{\%}^{\prime}\right)\right)$-Homöomorphismus ist.

Sei $(E, A)$ ein ausgeglichener, absolutkonvexer Raum. Das Tensorprodukt von $n$ Faktoren $E$ sei $\otimes^{n} E$ und die projektive, absolutkonvexe Tensorproduktlimitierung auf $\otimes^{n} E$ sei mit $\otimes_{k}^{n} A$ bezeichnet. Wir setzen $\left(\otimes^{0} E, \otimes_{k}^{0} A\right)$ gleich $\left(\mathbf{K}, \Lambda_{0}\right)$, wo $\Lambda_{0}$ die natürliche Topologie auf $\mathbf{K}$ ist $\left(A_{0}\right.$ ist ja die feinste Vektorraumlimitierung auf $\mathbf{K}$; siehe [1] und [10]). 
Auf der Tensoralgebra $\otimes E=\oplus_{n \in \mathbf{N}} \otimes^{n} E$, wo $\mathbf{N}$ die Menge $\{0,1,2, \ldots\}$ bezeichnet, sei $\otimes_{k} \Lambda$ die Limitierung $\oplus_{n \in \mathrm{N}} \otimes_{k}^{n} \Lambda$. Der Raum $\otimes_{k} E=$ $\left(\otimes E, \otimes_{k} \Lambda\right)$ ist eine Limesalgebra, denn die zu der kanonischen Multiplikation assoziierte lineare Abbildung ist stetig, wegen des Homöomorphismus $\left(\otimes_{k} E\right) \otimes_{k}\left(\otimes_{k} E\right) \rightarrow \oplus\left(\otimes_{k}^{n+m} E\right) \quad$ (Sätze 16, 4, 2, Bemerkung nach Satz 14).

Folgendes gilt (vgl. [9], Th. 3.1):

Satz 17. (1) Für jeden ausgeglichenen, absolutkonvexen Limesvektorraum $(E, A)$ ist $\left(\otimes E, \otimes_{k} \Lambda\right)$ eine ausgeglichene, absolutkonvexe Limesalgebra. Falls $E \neq\{0\}$ ist, so gibt es keine Vektorraumtopologie auf $\otimes E$, die feiner als $\otimes_{k} \Lambda$ ist.

(2) Für jede lineare Abbildung $f: E \rightarrow A$ von einem ausgeglichenen, absolutkonvexen Raum $(E, A)$ nach einer absolutkonvexen, assoziativen, unitären Limesalgebra $\left(A, \Lambda_{A}\right)$ ist $f$ genau dann $\left(\Lambda, \Lambda_{A}\right)$-stetig, wenn der von $f$ definierte Algebra-Homomorphismus $f \otimes: \otimes E \rightarrow A \quad\left(\otimes_{k} A, \Lambda_{A}\right)$ stetig ist. Die Limitierung $\otimes_{k} \Lambda$ ist also die feinste absolutkonvexe AlgebraLimitierung auf $\otimes E$, für die die natürtiche Injektion $E \rightarrow \otimes E$ stetig ist.

Beweis. (1) Nach 2.2.16 in [8] gibt es keine Vektorraumlimitierung feiner als $\otimes_{k} A$, falls $E \neq\{0\}$ ist. (2) folgt aus Satz 14, Kor. 2 und aus der Tatsache, dass der von der natürlichen Injektion $E \rightarrow \otimes E$ definierte Algebra-Homomorphismus die Identität auf $\otimes E$ ist.

Åbo Akademi

Mathematisches Institut

SF-20500 Åbo 50

Finnland 


\section{Literatur}

[1] Binz, E.: Ein Differenzierbarkeitsbegriff in limitierten Vektorräumen. - Comment. Math. Helv. 41, 137-156 (1966).

[2] -》- Keller, H. H.: Funktionenräume in der Kategorie der Limesräume. Ann. Acad. Sci. Fenn. A. I. 383 (1966).

[3] BJon, S.: Über absolutkonvexe Limesvektorräume. - Soc. Sci. Fenn. Comment. Phys.-Math. 43, 181-188 (1973).

[4] -»- Eine ausgeglichene Limitierung auf Räumen n-linearer Abbildungen zwischen Limesvektorräumen. - Soc. Sci. Fenn. Comment. Phys.-Math. 43, 189-201 (1973).

[5] Fischer, H. R.: Limesräume. - Math. Ann. 137, 269-303 (1959).

[6] Frölicher, A., Bucher, W.: Calculus in vector spaces without norm. - Lecture Notes in Mathematics 30. Springer-Verlag 1966.

[7] Grothendieck, A.: Produits tensoriels topologiques et espaces nucléaires. Mem. Amer. Math. Soc. Nr. 16 (1955).

[8] JARchow, H.: Marinescu-Räume. - Comment. Math. Helv. 44, 138-163 (1969).

[9] -»- On tensor algebras of Marinescu spaces. - Math. Ann. 187, 163-174 (1970).

[10] Kutzler, K.: Eine Bemerkung über endlichdimensionale, separierte, limitierte Vektorräume. - Arch. Math. (Basel) 20, 165-168 (1969).

[11] -»- Bemerkungen über unendlichdimensionale, separierte Limesvektorräume und Limesgruppen. - J. reine angew. Math. 253, 98-116 (1972).

[12] Wingres, C.-E.: Locally convex limit spaces. - Acta Acad. Aboensis, Ser. B 33 nr 5 (1973). 